\title{
Maintenance and Intensification of Bivalent Oral Poliovirus Vaccine Use Prior to its Coordinated Global Cessation
}

Radboud J Duintjer Tebbens ${ }^{1 *}$, Lee M Hampton ${ }^{2}$, Steven G F Wassilak ${ }^{2}$, Mark A Pallansch ${ }^{3}$, Stephen L Cochi ${ }^{2}$ and Kimberly M Thompson ${ }^{1}$

${ }^{1}$ Kid Risk, Inc., 10524 Moss Park Rd., Ste. 204-364, Orlando, FL 32832, USA

${ }^{2}$ Global Immunization Division, Center for Global Health, Centers for Disease Control and Prevention, Atlanta, GA, USA

${ }^{3}$ Division of Viral Diseases, National Center for Immunization and Respiratory Diseases, Centers for Disease Control and Prevention, Atlanta, GA, USA

\begin{abstract}
Objective: To examine the impact of different bivalent oral poliovirus vaccine (bOPV) supplemental immunization activity (SIA) strategies on population immunity to serotype 1 and 3 poliovirus transmission and circulating vaccinederived poliovirus (cVDPV) risks before and after globally-coordinated cessation of serotype 1 and 3 oral poliovirus vaccine (OPV13 cessation).
\end{abstract}

Methods: We adapt mathematical models that previously informed vaccine choices ahead of the trivalent oral poliovirus vaccine to bOPV switch to estimate the population immunity to serotype 1 and 3 poliovirus transmission needed at the time of OPV13 cessation to prevent subsequent cVDPV outbreaks. We then examine the impact of different frequencies of SIAs using bOPV in high risk populations on population immunity to serotype 1 and 3 transmission, on the risk of serotype 1 and 3 cVDPV outbreaks, and on the vulnerability to any imported bOPVrelated polioviruses

Results: Maintaining high population immunity to serotype 1 and 3 transmission using bOPV SIAs significantly reduces 1 ) the risk of outbreaks due to imported serotype 1 and 3 viruses, 2) the emergence of indigenous cVDPVs before or after OPV13 cessation, and 3) the vulnerability to bOPV-related polioviruses in the event of nonsynchronous OPV13 cessation or inadvertent bOPV use after OPV13 cessation.

Conclusion: Although some reduction in global SIA frequency can safely occur, countries with suboptimal routine immunization coverage should each continue to conduct at least one annual SIA with bOPV, preferably more, until global OPV13 cessation. Preventing cVDPV risks after OPV13 cessation requires investments in bOPV SIAs now through the time of OPV13 cessation.

Keywords: Polio; Eradication; Risk management; OPV; Vaccine choice

\begin{abstract}
Abbreviations: bOPV: bivalent oral poliovirus vaccine; $\operatorname{cVDPV}(1,2,3)$ : circulating VDPV (serotype 1,2 , or 3 , respectively); DEB: differential-equation based; EIP*: threshold effective immune proportion; EIPM: age-and-sub-population-mixing-adjusted effective immune proportion; IPV: inactivated poliovirus vaccine; iVDPV: immunodeficiency-associated VDPV; mOPV: monovalent oral poliovirus vaccine; OPV: oral poliovirus vaccine; OPV\#\#: globallycoordinated cessation of OPV containing the serotypes indicated by \#\#; $\mathrm{R}_{0}$ : basic reproduction number; $\mathrm{RI}$ : routine immunization; $\mathrm{R}_{\mathrm{n}}$ : mixingadjusted net reproduction number; SIA: supplemental immunization activity; tOPV: trivalent oral poliovirus vaccine; VDPV: vaccinederived poliovirus; $\mathrm{WPV}(1,2,3)$ : wild poliovirus (serotype 1, 2, or 3, respectively)
\end{abstract}

\section{Introduction}

Pakistan, Afghanistan, and Nigeria remain the only three countries with continued indigenous transmission of wild poliovirus (WPV) serotype 1 (WPV1). The last reported case associated with naturallyoccurring serotype 2 WPV (WPV2) occurred in 1999 [1], with certification of its global eradication in 2015 [2]. The last reported case associated with serotype 3 WPV (WPV3) occurred in 2012 [3], Oral poliovirus vaccine (OPV) cessation represents an essential part of the polio endgame because of the risks of paralytic poliomyelitis disease (polio) associated with continued use of the vaccine [4]. These risks include vaccine-associated paralytic polio in recipients and close contacts; the acquisition of WPV-like properties by OPV-related viruses as they continue to circulate in vulnerable populations (i.e., circulating vaccine-derived polioviruses (cVDPVs)); and long-term infections in rare individuals with B-cell related primary immunodeficiency diseases (i.e., immunodeficiency-associated vaccine-derived polioviruses (iVDPVs)) [4,5]. Between mid-April and early May 2016, 155 countries successfully coordinated the global switch from OPV containing attenuated strains of all three poliovirus serotypes (i.e. trivalent OPV (tOPV)), to bivalent OPV (bOPV) containing only serotypes 1 and 3 [6]. With the switch, the global cessation of serotype 2-containing OPV (OPV2) became a reality, and, if successful, will pave the way for the eventual cessation of the remaining two serotypes [7]. The current plan calls for simultaneous cessation of the remaining OPV serotypes (OPV13 cessation) [7], although additional delays in achieving WPV1 eradication could motivate earlier certification of WPV3 eradication and a switch from bOPV to monovalent OPV (mOPV) serotype $1[8,9]$

Due to the limited intestinal immunity provided by the inactivated poliovirus vaccine (IPV) [10-13], the addition of IPV to routine immunization (RI) schedules does not provide much reduction in cVDPV risks in populations at highest risk of such outbreaks [14-16].

*Corresponding author: Radboud J Duintjer Tebbens, Kid Risk, Inc., 10524 Moss Park Rd., Ste. 204-364, Orlando, FL 32832, USA, Tel: 781-325 8418; E-mail: rdt@kidrisk.org

Received September 15, 2016; Accepted September 28, 2016; Published October 03, 2016

Citation: Duintjer Tebbens RJ, Hampton LM, Wassilak SGF, Pallansch MA Cochi SL, et al. (2016) Maintenance and Intensification of Bivalent Oral Poliovirus Vaccine Use Prior to its Coordinated Global Cessation. J Vaccines Vaccin 7: 340 doi: $10.4172 / 2157-7560.1000340$

Copyright: (c) 2016 Duintjer Tebbens RJ, et al. This is an open-access article distributed under the terms of the Creative Commons Attribution License, which permits unrestricted use, distribution, and reproduction in any medium, provided the original author and source are credited. 
However, IPV provides the only source of individual immunity to polio (disease) after OPV cessation and over time provides populations with more protection from poliovirus transmission compared to no IPV. Populations in which conditions favor a greater contribution of oropharyngeal transmission of poliovirus compared to fecal-oral transmission (i.e., better hygiene and sanitation and more temperate climates) coincide with settings with higher expected prevalence of long-term iVDPV excretors [17]. In those populations, IPV provides more protection from poliovirus transmission because IPV provides better protection from oropharyngeal excretion compared to fecal excretion $[12,18]$.

In the context of preparing for the tOPV to bOPV switch, several mathematical modeling analyses demonstrated the need to intensify tOPV use prior to the switch to maximize population immunity to transmission (i.e., the aggregated ability of all individuals in a population to contribute to poliovirus transmission, including from individuals immune to polio (disease) that may participate in transmission asymptomatically [19]) for serotype 2. Doing so will minimize the risk of 1) continued circulation of indigenous serotype 2 cVDPV (cVDPV2) outbreaks that began before the switch [14,20,21], 2) continued transmission and evolution of OPV2-related viruses after the switch [14, 20], 3) cVDPV2 outbreaks due to importation of OPV2related poliovirus into countries that already switched from countries yet to switch in the event of a non-synchronous switch [22], and 4) cVDPV2 outbreaks following inadvertent tOPV [23] or deliberate serotype $2 \mathrm{mOPV}$ use after the switch [24].

With the tOPV intensification that preceded the now completed switch, WPV1 confined to three countries, and IPV introduced in all countries that experienced WPV or cVDPV outbreaks since 2000, questions emerge regarding the need to continue to conduct frequent supplemental immunization activities (SIAs) with bOPV in polio nonendemic countries to maintain high population immunity to serotype 1 and 3 transmission. This analysis uses the poliovirus transmission and OPV evolution models $[25,26]$ previously used to inform risk management of the tOPV to bOPV switch $[14,20,22,23]$ to investigate the impact of various levels of bOPV use on the risk of indigenous cVDPV1 and cVDPV3 outbreaks before or after OPV13 cessation and on the time after OPV13 cessation until populations become vulnerable to transmission of OPV-related virus strains with different degrees of reversion. We further compare and discuss strategies that focus on continued bOPV maintenance versus bOPV intensification only shortly before OPV13 cessation.

\section{Methods}

We previously developed and extensively tested a deterministic differential equation-based poliovirus transmission and OPV evolution model (i.e., the DEB model) (see appendix A1 available at: http://www. kidrisk.org for details) [25-27]. The compartmental model divides a population by age groups and for each of the three serotypes by 8 immunity states, 5 waning stages (for individuals with actively acquired immunity through IPV vaccination or live poliovirus infection), and 6 fecal and 6 oropharyngeal infection stages (for individuals infected with a live poliovirus) subdivided into 20 OPV reversion stages (for infections tracing back to reversion stages (for infections tracing back to). The choice of age groups varies by modeled population (e.g., to accommodate different RI schedules), although for all populations we assume preferential mixing within the three mixing age groups of 0-4 year olds, 5-14 year olds, and those age 15 years or older. The basic reproduction number $\left(\mathrm{R}_{0}\right)$ characterizes the inherent ability of a poliovirus to spread in a given population [28]. We assume fixed relative $R_{0}$ values between the three WPV serotypes (i.e., 1:0.9:0.75 for WPV1:WPV2:WPV3) and use the WPV1 $\mathrm{R}_{0}$ as an overall indicator of conditions that affect poliovirus transmissibility in a population (e.g., crowding, hygiene, sanitations, climate), which typically varies over time due to seasonality.

The subdivision by reversion stage provides an approximation of the process by which the attenuated polioviruses found in OPV evolve into VDPVs, characterized by serotype-specific average times to reach the last reversion stage (i.e., 408 days for serotype 2 and 621 days for serotype 1 and 3 ). The last reversion stage represents fully-reverted vaccine-derived poliovirus with the same assumed $R_{0}$ and paralysis-toinfection ratio as homotypic WPVs. The starting relative $\mathrm{R}_{0}$ values (i.e., 0.37 for OPV1, 0.55 for OPV2 and 0.25 for OPV3) and paralysis-toinfection ratios compared to homotypic WPV also vary by serotype, with the $\mathrm{R}_{0}$ and natural logarithm of the paralysis-to-infection ratio both increasing linearly by reversion stage (see appendix A1). The OPV evolution process simulates die-out of poliovirus transmission of any serotype and reversion stage (i.e., strain) in the (deterministic) DEB model once the prevalence of a given strain drops below the transmission threshold value of 5 per million people in any given mixing age group. If this occurs, we set the corresponding force-ofinfection to zero in the simulation, such that no new infections occur for the serotype and reversion stage. Conversely, viruses only begin to circulate at a given reversion stage once the prevalence exceeds the transmission threshold after sufficient inflow from lower reversion stages. The OPV evolution process in the model adequately reproduced secondary OPV transmission and the occurrence or absence of cVDPV outbreaks in a diverse set of populations $[12,25,29,30]$, We observe cVDPV emergences in the DEB model (i.e. defined as the occurrence of prevalence of virus in the last reversion stage above the transmission threshold) only when OPV-related viruses circulate in a population with low enough population immunity to sustain transmission $[15,16,20]$, consistent with the evidence from cVDPV outbreaks [31-33].

We characterize population immunity to transmission in the model using the mixing-adjusted effective immune proportion (EIPM), which aggregates the relative potential to participate in transmission of all individuals compared to fully susceptible individuals and also takes into account the mixing structure between different age groups and any preferentially mixing subpopulations [16]. An EIPM of 0 indicates that all individuals in a given population are completely susceptible to transmission, and higher values indicate higher effective proportions of the population with immunity that provides protection from reinfection and/or poliovirus excretion. Although we assume full immunity from polio (disease) for all successfully vaccinated or previously infected individuals, these immune individuals may still participate asymptomatically in transmission to varying degrees. The EIPM relates closely to the net reproduction number $\left(R_{n}\right.$, equal to $\left.[1-E I P M] \times R_{0}\right)$, which represents the average number of secondary infections generated by a new infection given the existing population immunity to transmission. Given that $\mathrm{R}_{\mathrm{n}}$ depends directly on $\mathrm{R}_{0,}$ a given EIPM implies different $R_{n}$ values for strains with different $R_{0}$, values. Transmission of a given strain will eventually die out if its $R_{n}$ remains less than 1. Therefore, in the context of absence of any indigenous poliovirus circulation, we use the occurrence of an $\mathrm{R}_{\mathrm{n}}$ greater than 1 for a given strain as an indicator of vulnerability of a given population to circulation of that strain if that strain were imported into that population. The corresponding threshold effective immune proportion (i.e., $\mathrm{EIP}^{*}=1-1 / \mathrm{R}_{0}$ ) differs by strain because it depends on $\mathrm{R}_{0}$ (i.e., a strain with a higher $\mathrm{R}_{0}$ can circulate at a higher EIPM). Our analysis 
focuses on characterizing the vulnerability of populations to potential importations as a result of different bOPV strategies, but does not model actual importations or their consequences, which represent random events that could alter vulnerability going forward in the event of widespread circulation and/or outbreak response immunization.

Table 1 shows the population-specific inputs for a hypothetical population (i.e., high $\mathrm{R}_{0}$, no seasonality) as well as three populations based on the populations of northern India, northern Pakistan and Afghanistan, and northern Nigeria that we previously considered for analyses of the risks associated with potential non-synchronous OPV2 cessation [22], or inadvertent tOPV use after OPV2 cessation [23]. The populations like northern India, northern Pakistan and Afghanistan, and northern Nigeria represent selections from a larger global longterm poliovirus risk management model [34]. The properties include those related to inherent transmissibility of polioviruses (i.e., WPV $\mathrm{R}_{0}$ and seasonality, role of oropharyngeal transmission), RI coverage, SIA impact (i.e., true coverage and repeated missed probability, defined as the "conditional probability that a targeted individual does not receive a dose in a round, given that the individual did not receive a dose in the previous round despite falling into the targeted population for that round" [26]), and surveillance (not used in this paper because we did not consider outbreak response). All populations assume the same generic DEB model inputs $[25,26]$ and simplified vaccination histories, including IPV administration with the third non-birth OPV dose from January 1, 2015 [34] and tOPV intensification ahead of the tOPV to bOPV switch on May 1, 2016. They further assume administration of a birth dose of OPV with $50 \%$ of the coverage achieved with 3 non-birth RI doses (POL3); that $20 \%$ of children who do not receive at least 3 non-birth RI doses (i.e., 100\%-POL3) receive 1 dose; and that another $20 \%$ of children who do not receive at least 3 non-birth RI doses receive 2 doses, based on typical values for low- and lower middle-income countries $[25,34,35]$. We also consider a hypothetical population with a WPV1 $\mathrm{R}_{0}$ of 13 and no seasonality to control for any seasonalityassociated behavior, and which otherwise assumes the same properties as the under-vaccinated northern Indian population.

For both the hypothetical and realistic populations, the first analysis uses the DEB model to determine the relationship between RI coverage and population immunity and the occurrence of indigenous cVDPV outbreaks before or after OPV13 cessation for serotypes 1 and 3. For this analysis, we assume no SIAs for long enough (i.e., 9 years) prior to OPV13 cessation to achieve approximately constant population immunity at the time of OPV 13 cessation. This analysis follows the same approach as a prior analysis of the relationship between RI coverage with tOPV and cVDPV2 risks after OPV2 cessation [20]. However, the assumptions of this analysis differ from that of the prior analysis (even for serotype 2 polioviruses) because of the inclusion of the birth OPV dose, partial coverage with 1 or 2 OPV doses, and the introduction of IPV in 2015.

We use the same hypothetical population to illustrate the impact of the annual number of bOPV SIAs on population immunity to serotype 1 and 3 transmission over time and on OPV13 cessation dynamics. This analysis quantifies the impact of the annual number of bOPV SIAs on die-out of OPV-related viruses after OPV13 cessation and on the vulnerability to different serotype 1 and 3 strains for the realistic northern India, northern Pakistan and Afghanistan, and northern Nigeria modeled populations. To control for the timing of the last bOPV SIA before OPV13 cessation and to ensure that all bOPV SIAs occur before OPV 13 cessation, this analysis assumes 4 annual bOPV SIAs that occur on days $0,40,80$, and 120 of each year, 3 annual bOPV SIAs that occur on days 40, 80, and 120, 2 annual SIAs on days 80 and 120,1 annual SIA on day 120, or no SIAs.

In an additional analysis to explore the role of IPV, we repeated the above runs but without any IPV use in RI and compared this to the results with IPV in RI from 2015 on. Finally, we model the realistic populations to explore the population immunity and indigenous cVDPV risk implications of maintaining bOPV SIAs up until the time of OPV13 cessation versus no bOPV SIAs during 2017-2019 followed by bOPV intensification in early 2020 shortly before OPV 13 cessation. All analyses assume OPV13 cessation on May 1, 2020 and exclude any immunization response to CVDPV outbreaks.

\section{Results}

Figure 1a shows that for the modeled hypothetical population without seasonality, no indigenous cVDPV1 or cVDPV3 outbreaks occur before or after OPV13 cessation when RI coverage exceeds $65 \%$. The results shown in Figure 1a also indicate the significant drop in population immunity to transmission following successfully coordinated OPV13 cessation in this hypothetical population. The EIP* needed to prevent cVDPV circulation differs by serotype because we assume that the $\mathrm{R}_{0}$ values of WPVs and fully reverted VDPVs vary by

\begin{tabular}{|c|c|c|c|c|c|c|c|c|c|}
\hline Population with properties like & $\begin{array}{c}\text { WPV1 } \\
\mathrm{R}_{0}{ }^{\mathrm{a}}\end{array}$ & $\begin{array}{c}\mathbf{R}_{0} \\
\text { amplitude }^{b}\end{array}$ & $\begin{array}{c}\text { Peak } \\
\text { day }\end{array}$ & $\begin{array}{l}\text { Degree of age- } \\
\text { heterogeneous } \\
\text { mixing }(k)^{c}\end{array}$ & $\begin{array}{c}\text { Average per- } \\
\text { dose bOPV } \\
\text { take rate }^{d}\end{array}$ & $\begin{array}{c}\text { Routine im- } \\
\text { munization } \\
\text { coverage } \\
\text { (POL3) }\end{array}$ & $\begin{array}{c}\text { True SIA } \\
\text { cover- } \\
\text { age }\end{array}$ & $\begin{array}{l}\text { Repeated } \\
\text { missed } \\
\text { probability }\end{array}$ & $\begin{array}{c}\text { Proportion of } \\
\text { transmissions } \\
\text { oropharyngeal } \\
\left(p^{\text {oro }}\right)\end{array}$ \\
\hline Hypothetical (no seasonality) & 13 & 0 & N/A & 0.35 & 0.45 & 0.3 & 0.8 & 0.7 & 0.3 \\
\hline Northern India, general & 13 & 0.2 & 180 & 0.35 & 0.45 & 0.6 & 0.95 & 0.5 & 0.3 \\
\hline Northern India, under-vaccinated & 13 & 0.2 & 180 & 0.35 & 0.45 & 0.3 & 0.8 & 0.7 & 0.3 \\
\hline Northern Pakistan and Afghanistan, general & 11 & 0.2 & 180 & 0.35 & 0.5 & 0.6 & 0.8 & 0.7 & 0.3 \\
\hline $\begin{array}{l}\text { Northern Pakistan and Afghanistan, under- } \\
\text { vaccinated }\end{array}$ & 11 & 0.2 & 180 & 0.35 & 0.5 & 0.1 & 0.3 & 0.95 & 0.3 \\
\hline Northern Nigeria, general & 8 & 0.1 & 120 & 0.4 & 0.54 & 0.3 & 0.5 & 0.8 & 0.3 \\
\hline Northern Nigeria, under-vaccinated & 8 & 0.1 & 120 & 0.4 & 0.54 & 0.05 & 0.15 & 0.95 & 0.3 \\
\hline
\end{tabular}

${ }^{a} R_{0}$ for WPV3 assumed equal to 0.75 times $R_{0}$ for WPV 1

bDefined as the "proportional change in $R_{0}$ due to seasonality" [25]

"Defined as the "proportion of contacts reserved for individuals within the same mixing age group" [25]

Defined as the proportion of vaccine recipients that develops an immune response

eDefined as the coverage with 3 or more non-birth routine immunization doses

fDefined as the conditional probability that a targeted individual does not receive a dose in a round, given that the individual did not receive a dose in the previous round despite falling into the targeted population for that round" [26]

Table 1: Population-specific characteristics of populations considered for analysis [22,23,34], based on previously characterized populations [25,26] 

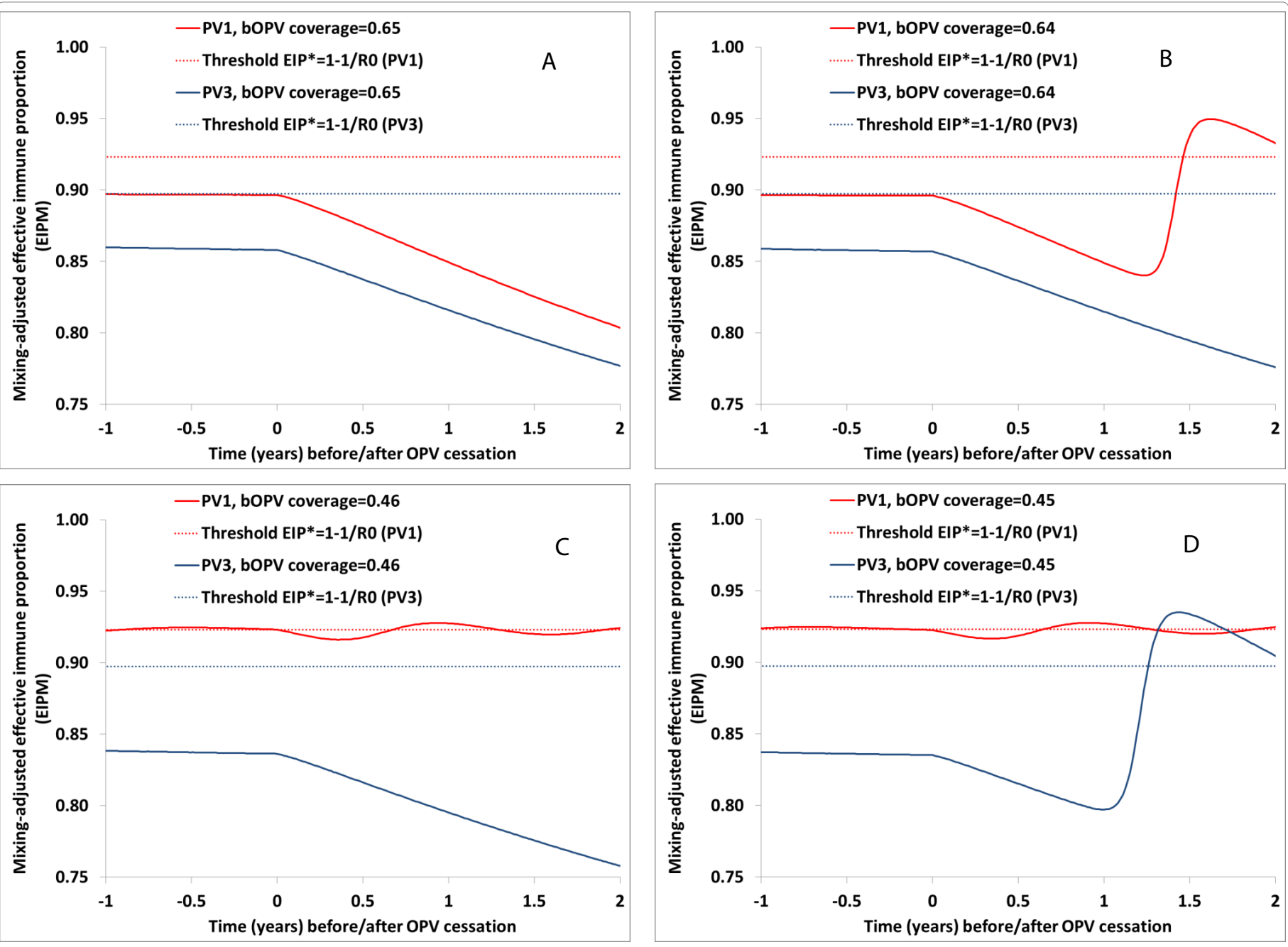

Figure 1: Population immunity to WPV1 and WPV3 transmission before and after OPV13 cessation for different routine immunization coverage with 3 non-birth bOPV doses (including IPV co-administration at third dose) in the hypothetical population with an R0 for WPV1 equal to $13^{*}$. Increasing population immunity reflects occurrence of an indigenous cVDPV outbreak. A) Lowest coverage that prevents both cVDPV1 and cVDPV3 outbreaks. B) Highest coverage for which a cVDPV1 outbreak occurs (but no cVDPV3 outbreak). C) Lowest coverage that prevents a cVDPV3 outbreak (but which does not prevent a pre-cessation cVDPV1 outbreak). D) Highest coverage for which both a cVDPV1 and a cVDPV3 outbreak occur. ${ }^{*} R_{0}$ for WPV3 $=0.75 \times R_{0}$ for WPV1 (i.e., $0.75 \times 13=9.75$ )

serotype $[25,26]$. Despite the same bOPV take rate (i.e., the proportion of vaccine recipients that develops an immune response) for serotypes 1 and 3 (Table 1), we find higher EIPM for serotype 1 than serotype 3 due to the greater secondary spread for serotype 1 as a result of the higher assumed $\mathrm{R}_{0}$ values for viruses closely related to OPV1 compared to viruses closely related to OPV3. In this model of a hypothetical population, highly-reverted polioviruses do not already circulate at the time of OPV13 cessation due to sufficient population immunity to prevent extensive circulation and evolution of lower reversion stage viruses, which die out after OPV13 cessation. Therefore, successful OPV13 cessation for both serotypes can occur even with population immunity to transmission below the respective EIP*. However, any further decrease in bOPV coverage and resulting decrease in population immunity to transmission allows continued circulation and evolution of the existing OPV1-related viruses after OPV13 cessation, which results in a cVDPV1 outbreak in the hypothetical population shown by the increasing EIPM in the second year after OPV13 cessation in Figure 1b. However, for the same RI coverage no cVDPV3 outbreak occurs because of the low transmissibility of viruses closely related to OPV3.
We find that for RI coverage of $46 \%$, no cVDPV3 outbreak occurs (Figure 1c) even though for such low coverage a cVDPV1 outbreak would occur 5 years before OPV13 cessation in the hypothetical population (leading the EIPM to oscillate around the threshold at the time of OPV cessation in the absence of an outbreak response, as shown Figure 1c). RI coverage below $46 \%$ results in both an indigenous cVDPV3 outbreak after OPV13 cessation and an indigenous cVDPV1 outbreak well before OPV13 cessation (Figure 1d).

Comparing the minimum population immunity to poliovirus transmission to prevent a cVDPV after OPV cessation of each serotype in the hypothetical population, and using separate model runs for each serotype with different coverage levels, Figure 2 shows that preventing cVDPV2 outbreaks after OPV2 cessation in the hypothetical population requires the highest population immunity to transmission. This remains consistent with the known rapid reversion and high transmissibility of the attenuated serotype 2 poliovirus in OPV $[32,36,37]$ and the corresponding model assumptions of short reversion times and high relative $\mathrm{R}_{0}$ values for serotype 2 . Preventing a cVDPV1 outbreak after OPV1 cessation requires somewhat lower population 
Citation: Duintjer Tebbens RJ, Hampton LM, Wassilak SGF, Pallansch MA, Cochi SL, et al. (2016) Maintenance and Intensification of Bivalent Oral Poliovirus Vaccine Use Prior to its Coordinated Global Cessation. J Vaccines Vaccin 7: 340. doi: 10.4172/2157-7560.1000340

Page 5 of 9

immunity to transmission than preventing a cVDPV2 outbreak after OPV2 cessation. Preventing a cVDPV3 outbreak after OPV3 cessation requires even lower population immunity to transmission. The arrows in Figure 2 show that for serotype 2, population immunity to transmission at OPV2 cessation must remain at its threshold of approximately 0.91 in this hypothetical population model to prevent a subsequent cVDPV2 outbreak, while it can remain somewhat lower than the threshold for serotype 1 and even lower for serotype 3 without subsequent cVDPV1 and cVPDV3 outbreaks. However, with respect to the minimum RI coverage needed to prevent cVDPV outbreaks after OPV cessation, we find that successful serotype 1 cessation requires the highest RI coverage, followed by serotype 2 cessation and serotype 3 cessation (see Figure 2 legend for RI coverage values). The need for relatively higher RI coverage for successful serotype 1 compared to serotype 2 cessation relates primarily to greater secondary exposure to serotype 2 than serotype $1 \mathrm{OPV}$-related viruses that provides or boosts

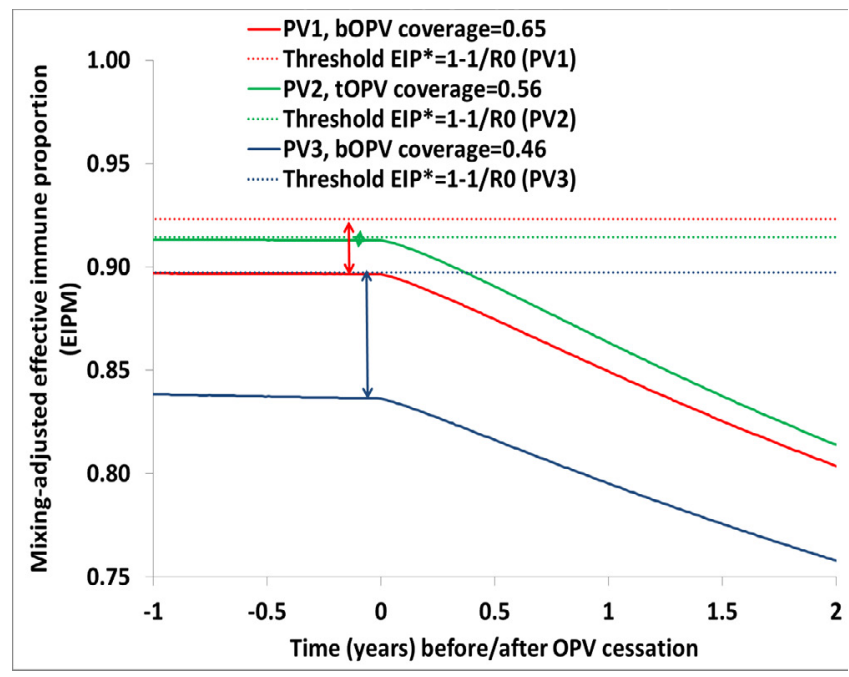

Figure 2: Minimum population immunity to transmission of each WPV serotype at tOPV or OPV13 cessation to prevent a subsequent homotypic cVDPV outbreak in the hypothetical population with an $R_{0}$ for WPV1 equal to $13^{*}{ }^{*} R_{0}$ for $W P V 2=0.9 \times R_{0}$ for WPV1 (i.e., $0.9 \times 13=11.7$ ) and $R_{0}$ for $W P V 3=0.75 \times R_{0}$ for WPV1 (i.e., $0.75 \times 13=9.75$ ) immunity of individuals who come into contact with OPV recipients [36,37].

While Figures 1 and 2 considered the effect of RI only in the hypothetical population, Figure 3 shows the population immunity to transmission and OPV13 cessation dynamics as a function of the annual number of bOPV SIAs in the hypothetical population with a WPV1 $\mathrm{R}_{0}$ of 13 . This clearly demonstrates the need to continue some level of bOPV SIAs to prevent indigenous CVDPV outbreaks in this type of population. Figure 3 shows that in the absence of bOPV SIAs, cVDPV outbreaks occur before OPV13 cessation for serotype 1 (Figure 3a) and after OPV13 cessation for serotype 3 (Figure 3b). With at least one annual bOPV SIA, $\mathrm{R}_{\mathrm{n}}$ values of fully-reverted VDPV and WPV oscillate around or over 1 , which remains sufficient to prevent indigenous cVDPV 1 and cVDPV 3 emergence, but which implies the possibility of widespread circulation in the event of an importation of WPV1, cVDPV1, or cVDPV3 from another population. Due to the lower $\mathrm{R}_{0}$ of WPV 3 compared to WPV1 (i.e., WPV $3 \mathrm{R}_{0}=0.75 \times \mathrm{WPV} 1$ $R_{0}$ ), the same bOPV frequency leads to somewhat lower $R_{n}$ values for serotype 3 compared to serotype 1 despite the lower secondary spread of OPV3-related viruses compared to OPV1-related viruses.

Table 2 shows how the bOPV SIA frequency affects die-out of OPV1- and OPV3-related viruses after OPV13 cessation for all modeled populations. A consistent pattern emerges of longer times after OPV13 cessation until OPV-related viruses die out for lower bOPV SIA frequencies. Lowering the bOPV SIA frequency first results in an indigenous cVDPV1 outbreak after OPV13 cessation and eventually can lead to a cVDPV1 outbreak before OPV13 cessation and a cVDPV3 outbreak after OPV13 cessation. The frequency with which this occurs depends on the characteristics of the population, including the $\mathrm{R}_{0}$ value for the population and the RI coverage level (Table 1). For populations with lower $\mathrm{R}_{0}$ values (e.g., like northern Nigeria), low RI coverage may sustain sufficient population immunity to prevent indigenous cVDPV outbreaks, particularly for serotype 3 due the low transmissibility of viruses closely related to OPV3.

Based on the same model runs as Table 2 and Table 3 show how long it takes before the different populations become vulnerable to transmission of any imported OPV1- and OPV3-related viruses (i.e., attain an $\mathrm{R}_{\mathrm{n}}>1$ ) after OPV13 cessation (e.g., due to non-synchronous
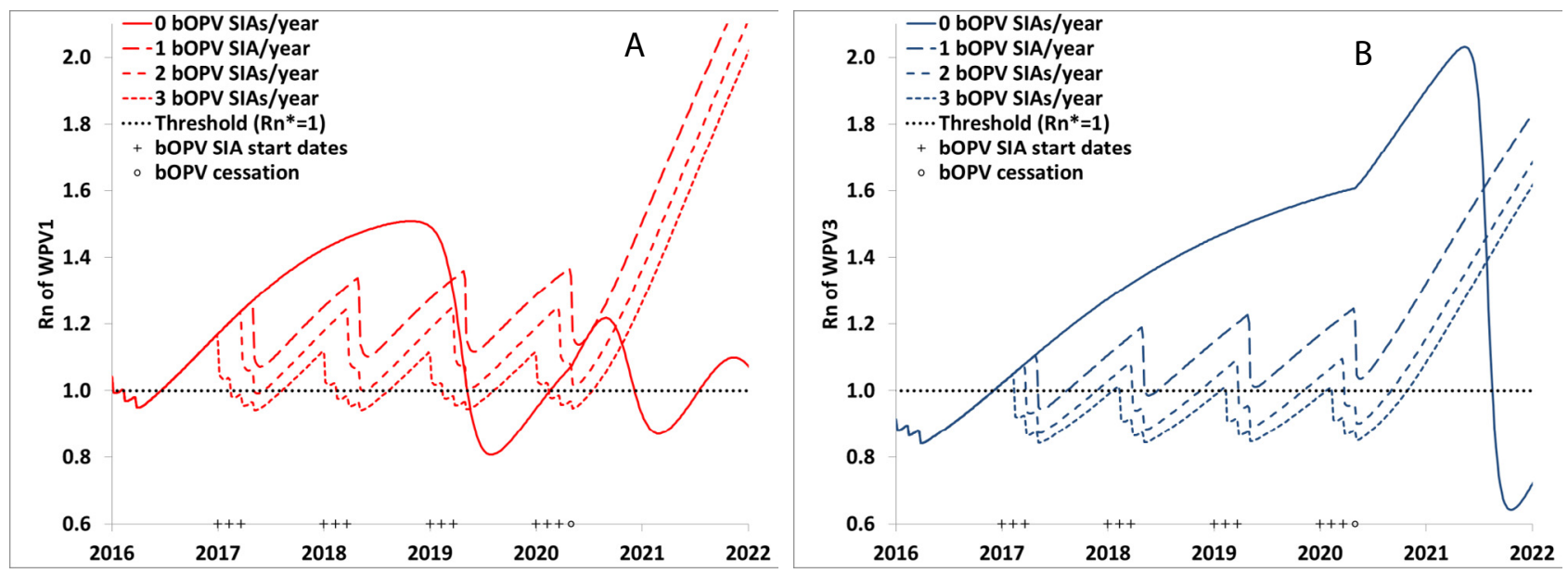

Figure 3: Population immunity to transmission as a function of the annual number of bOPV SIAs in the hypothetical population with a R0 for WPV1 equal to 13 . A) Serotype 1. B) Serotype 3. 
Citation: Duintjer Tebbens RJ, Hampton LM, Wassilak SGF, Pallansch MA, Cochi SL, et al. (2016) Maintenance and Intensification of Bivalent Oral Poliovirus Vaccine Use Prior to its Coordinated Global Cessation. J Vaccines Vaccin 7: 340. doi: 10.4172/2157-7560.1000340

Page 6 of 9

\begin{tabular}{|c|c|c|c|}
\hline \multirow{2}{*}{$\begin{array}{l}\text { Population with } \\
\text { properties like }\end{array}$} & \multirow{2}{*}{$\begin{array}{c}\text { Annual } \\
\text { \# bOPV SIAs }\end{array}$} & \multicolumn{2}{|c|}{$\begin{array}{c}\text { Time (days since OPV13 cessation) } \\
\text { until die-out of OPV-related virus }\end{array}$} \\
\hline & & Serotype 1 & Serotype 3 \\
\hline \multirow{5}{*}{$\begin{array}{l}\text { Hypothetical (no } \\
\text { seasonality), WPV1 } \\
\mathrm{R}_{0}=13\end{array}$} & 0 & $\begin{array}{l}\text { Pre-cessation } \\
\text { cVDPV }\end{array}$ & $\begin{array}{l}\text { Post-cessation } \\
\text { cVDPV }\end{array}$ \\
\hline & 1 & 190 & 135 \\
\hline & 2 & 130 & 105 \\
\hline & 3 & 115 & 95 \\
\hline & 4 & 105 & 95 \\
\hline \multirow{5}{*}{$\begin{array}{l}\text { Hypothetical (no } \\
\text { seasonality), WPV1 } \\
\mathrm{R}_{0}=10\end{array}$} & 0 & $\begin{array}{l}\text { Post-cessation } \\
\text { cVDPV }\end{array}$ & 120 \\
\hline & 1 & 150 & 125 \\
\hline & 2 & 115 & 100 \\
\hline & 3 & 105 & 95 \\
\hline & 4 & 95 & 90 \\
\hline \multirow{5}{*}{ Northern India, general } & 0 & $\begin{array}{l}\text { Post-cessation } \\
\text { cVDPV }\end{array}$ & 150 \\
\hline & 1 & 165 & 120 \\
\hline & 2 & 125 & 100 \\
\hline & 3 & 115 & 90 \\
\hline & 4 & 105 & 90 \\
\hline \multirow{5}{*}{$\begin{array}{l}\text { Northern India, under- } \\
\text { vaccinated }\end{array}$} & 0 & $\begin{array}{l}\text { Pre-cessation } \\
\text { cVDPV }\end{array}$ & $\begin{array}{l}\text { Post-cessation } \\
\text { cVDPV }\end{array}$ \\
\hline & 1 & 225 & 150 \\
\hline & 2 & 155 & 115 \\
\hline & 3 & 130 & 100 \\
\hline & 4 & 120 & 95 \\
\hline \multirow{5}{*}{$\begin{array}{l}\text { Northern Pakistan and } \\
\text { Afghanistan, general }\end{array}$} & 0 & 255 & 120 \\
\hline & 1 & 145 & 115 \\
\hline & 2 & 115 & 100 \\
\hline & 3 & 105 & 90 \\
\hline & 4 & 100 & 85 \\
\hline \multirow{5}{*}{$\begin{array}{l}\text { Northern Pakistan and } \\
\text { Afghanistan, under- } \\
\text { vaccinated }\end{array}$} & 0 & $\begin{array}{l}\text { Pre-cessation } \\
\text { cVDPV }\end{array}$ & $\begin{array}{l}\text { Post-cessation } \\
\text { cVDPV }\end{array}$ \\
\hline & 1 & $\begin{array}{l}\text { Post-cessation } \\
\text { cVDPV }\end{array}$ & 260 \\
\hline & 2 & $\begin{array}{l}\text { Post-cessation } \\
\text { cVDPV }\end{array}$ & 185 \\
\hline & 3 & 250 & 155 \\
\hline & 4 & 210 & 135 \\
\hline \multirow{5}{*}{$\begin{array}{l}\text { Northern Nigeria, } \\
\text { general }\end{array}$} & 0 & 190 & 110 \\
\hline & 1 & 145 & 120 \\
\hline & 2 & 120 & 105 \\
\hline & 3 & 105 & 95 \\
\hline & 4 & 100 & 90 \\
\hline \multirow{5}{*}{$\begin{array}{l}\text { Northern Nigeria, } \\
\text { under-vaccinated }\end{array}$} & 0 & $\begin{array}{l}\text { Pre-cessation } \\
\text { cVDPV }\end{array}$ & 190 \\
\hline & 1 & $\begin{array}{l}\text { Post-cessation } \\
\text { cVDPV }\end{array}$ & 180 \\
\hline & 2 & 260 & 155 \\
\hline & 3 & 195 & 135 \\
\hline & 4 & 170 & 125 \\
\hline
\end{tabular}

Table 2: Impact of the annual number of bOPV SIAs on the persistence of OPV1and OPV3-related polioviruses after OPV13 cessation in different populations.

OPV13 cessation or inadvertent bOPV use [22,23]) as a function of bOPV SIA frequency. Table 3 shows that higher bOPV SIA frequency consistently implies more time until the first countries become vulnerable to different OPV1- and OPV3-related strains. However, for the parent OPV1 and OPV3 strains (stage 0), the time until countries become vulnerable remains at least two years, at which point the effect of bOPV SIA frequency before OPV13 cessation becomes small. For stage 10 OPV1- and OPV3-related viruses, which may circulate in a population that still uses OPV and then enter another population through an importation [22], Table 3 shows a greater effect of bOPV SIA frequency, with one annual bOPV SIA in some cases leading to a difference of over a year until vulnerability (e.g., northern Nigeria general population, between 1, 2 annual bOPV SIAs). For cVDPV1 and cVDPV3 (assumed to behave the same as WPV1 and WPV3, respectively), we find an even greater effect of the number of bOPV SIAs. For these results, zeroes indicate that for the given bOPV frequency the population immunity to transmission has already become so low that the population can support transmission of an imported cVDPV1 or cVDPV3 before OPV13 cessation.

In an additional analysis of the role of IPV, we found that failure to introduce IPV increased vulnerability, particularly to lower reversion stage viruses for which vulnerability only occurs long after OPV13 cessation (see appendix A2). Moreover, failure to introduce IPV resulted in longer times until die-out of OPV1- and OPV3-related viruses. For two combinations of populations and bOPV frequencies (i.e., northern Pakistan and Afghanistan general population with 0 annual bOPV SIAs and northern Pakistan and Afghanistan undervaccinated subpopulation with 3 annual bOPV SIAs), not using IPV resulted in an indigenous cVDPV1 outbreak that would not occur with IPV introduced in 2015. This reflects a greater cumulative effect of IPV on population immunity to serotype 1 and 3 transmission between IPV introduction in 2015 and OPV13 cessation in 2020 compared to the previously estimated very limited effect of IPV use between IPV introduction in 2015 and OPV2 cessation in 2016 [14].

Table 4 compares a strategy of bOPV intensification in 2020 (i.e., between 1 and 4 bOPV SIAs in 2020 prior to OPV13 cessation without maintenance of bOPV SIAs between 2017 and 2019) with a strategy of annual maintenance using one or three annual bOPV SIAs. Intensification with bOPV prevents indigenous cVDPV1 and cVDPV3 outbreaks after OPV13 cessation in the three general populations in Table 4, regardless of the number of intensification SIAs, but in all three under-vaccinated subpopulations indigenous cVDPV1 outbreaks already occur before OPV13 cessation. Moreover, bOPV intensification results in substantially greater proportions of the three-year period 2017-2019 during which the $\mathrm{R}_{\mathrm{n}}$ values for cVDPV1 and cVDPV3 remain greater than 1 , which implies greater vulnerability to imported cVDPVs or WPVs. In the under-vaccinated subpopulations, high proportions with $\mathrm{R}_{\mathrm{n}}>1$ occur even with bOPV maintenance due to the low impact and high repeated missed probabilities of SIAs (Table 1), but bOPV intensification implies significantly greater vulnerability overall during 2017-2019. For example, in the under-vaccinated subpopulation in northern Nigeria, the average $\mathrm{R}_{\mathrm{n}}$ for cVDPV3 during 2017-2019 equals 1.14 with 3 annual bOPV SIAs, 1.26 with 1 annual bOPV SIA, and 1.36 without bOPV maintenance.

\section{Discussion}

As in the case of coordinated global OPV2 cessation, the best time to manage the risks of post-OPV13 cessation cVDPV1 and cVDPV3 outbreaks is between now and the time of OPV13 cessation. Preventing cVDPV 1 and cVDPV3 outbreaks before and after coordinated OPV13 cessation requires maintaining sufficiently high population immunity to transmission to stop OPV-related viruses introduced before OPV13 cessation from establishing chains of transmission and evolving to cVDPVs. Maintaining high population immunity to poliovirus transmission also plays a critical role in preventing outbreaks from importations of WPV1 $[27,38]$, which remain possible as long as WPV1 circulates anywhere and which can divert resources from, or delay, 
Citation: Duintjer Tebbens RJ, Hampton LM, Wassilak SGF, Pallansch MA, Cochi SL, et al. (2016) Maintenance and Intensification of Bivalent Oral Poliovirus Vaccine Use Prior to its Coordinated Global Cessation. J Vaccines Vaccin 7: 340. doi: 10.4172/2157-7560.1000340

Page 7 of 9

\begin{tabular}{|c|c|c|c|c|c|c|c|}
\hline \multirow{2}{*}{$\begin{array}{l}\text { Population with } \\
\text { properties like }\end{array}$} & \multirow{2}{*}{$\begin{array}{c}\text { Annual \# bOPV } \\
\text { SIAs }\end{array}$} & \multicolumn{6}{|c|}{ Time (days since OPV13 cessation) until $R_{n}>1$} \\
\hline & & OPV1 (stage 0) & OPV3 (stage 0) & $\begin{array}{l}\text { OPV1-related } \\
\text { stage } 10\end{array}$ & $\begin{array}{l}\text { OPV3-related } \\
\text { stage } 10\end{array}$ & VDPV1 (stage 19) & VDPV3 (stage 19) \\
\hline \multirow{5}{*}{$\begin{array}{l}\text { Hypothetical (no } \\
\text { seasonality), WPV1 } \\
\mathrm{R}_{0}=13\end{array}$} & 0 & $N A^{a}$ & NA & NA & NA & NA & NA \\
\hline & 1 & 890 & 4710 & 210 & 405 & 0 & 0 \\
\hline & 2 & 955 & 4875 & 280 & 520 & 0 & 125 \\
\hline & 3 & 990 & 4945 & 310 & 565 & 55 & 180 \\
\hline & 4 & 1005 & 4990 & 325 & 595 & 75 & 210 \\
\hline \multirow{5}{*}{$\begin{array}{l}\text { Hypothetical (no } \\
\text { seasonality), WPV1 } \\
\mathrm{R}_{0}=10\end{array}$} & 0 & NA & $>25 \mathrm{yrs}$ & NA & 235 & NA & 0 \\
\hline & 1 & 1475 & $>25$ yrs. & 365 & 715 & 70 & 165 \\
\hline & 2 & 1570 & $>25 \mathrm{yrs}$ & 455 & 840 & 170 & 295 \\
\hline & 3 & 1610 & $>25 \mathrm{yrs}$ & 495 & 900 & 215 & 355 \\
\hline & 4 & 1635 & $>25 \mathrm{yrs}$ & 515 & 930 & 235 & 385 \\
\hline \multirow{5}{*}{$\begin{array}{l}\text { Northern India, } \\
\text { general }\end{array}$} & 0 & NA & 2950 & NA & 35 & NA & 0 \\
\hline & 1 & 760 & 3315 & 325 & 390 & 0 & 0 \\
\hline & 2 & 785 & 3320 & 340 & 440 & 0 & 40 \\
\hline & 3 & 1070 & 3325 & 345 & 690 & 0 & 315 \\
\hline & 4 & 1070 & 3330 & 350 & 695 & 0 & 320 \\
\hline \multirow{5}{*}{$\begin{array}{l}\text { Northern India, under- } \\
\text { vaccinated }\end{array}$} & 0 & NA & NA & NA & NA & NA & NA \\
\hline & 1 & 735 & 2945 & 305 & 355 & 0 & 0 \\
\hline & 2 & 750 & 2955 & 320 & 385 & 0 & 0 \\
\hline & 3 & 760 & 2960 & 330 & 405 & 0 & 25 \\
\hline & 4 & 765 & 2960 & 335 & 420 & 0 & 40 \\
\hline \multirow{5}{*}{$\begin{array}{l}\text { Northern Pakistan and } \\
\text { Afghanistan, general }\end{array}$} & 0 & 745 & 3685 & 30 & 345 & 0 & 0 \\
\hline & 1 & 1085 & 4050 & 345 & 695 & 15 & 305 \\
\hline & 2 & 1090 & 4055 & 360 & 705 & 55 & 325 \\
\hline & 3 & 1095 & 4060 & 365 & 715 & 290 & 335 \\
\hline & 4 & 1095 & 4060 & 370 & 715 & 295 & 340 \\
\hline \multirow{5}{*}{$\begin{array}{l}\text { Northern Pakistan and } \\
\text { Afghanistan, under- } \\
\text { vaccinated }\end{array}$} & 0 & NA & NA & NA & NA & NA & NA \\
\hline & 1 & NA & 2955 & NA & 20 & NA & 0 \\
\hline & 2 & NA & 2965 & NA & 330 & NA & 0 \\
\hline & 3 & 740 & 2975 & 300 & 345 & 0 & 0 \\
\hline & 4 & 745 & 3310 & 305 & 350 & 0 & 0 \\
\hline \multirow{5}{*}{$\begin{array}{l}\text { Northern Nigeria, } \\
\text { general }\end{array}$} & 0 & 1415 & $>25 \mathrm{yrs}$ & 245 & 365 & 0 & 0 \\
\hline & 1 & 1770 & $>25 \mathrm{yrs}$ & 370 & 740 & 220 & 270 \\
\hline & 2 & 1780 & $>25 \mathrm{yrs}$ & 610 & 1015 & 260 & 315 \\
\hline & 3 & 1790 & $>25 \mathrm{yrs}$ & 625 & 1030 & 280 & 350 \\
\hline & 4 & 1795 & $>25 \mathrm{yrs}$ & 635 & 1040 & 290 & 390 \\
\hline \multirow{5}{*}{$\begin{array}{l}\text { Northern Nigeria, } \\
\text { under-vaccinated }\end{array}$} & 0 & NA & $>25 \mathrm{yrs}$ & NA & 0 & NA & 0 \\
\hline & 1 & NA & $>25 \mathrm{yrs}$ & NA & 290 & NA & 0 \\
\hline & 2 & 1400 & $>25 \mathrm{yrs}$ & 255 & 325 & 0 & 0 \\
\hline & 3 & 1410 & $>25 \mathrm{yrs}$ & 270 & 370 & 0 & 0 \\
\hline & 4 & 1415 & $>25 \mathrm{yrs}$ & 280 & 635 & 0 & 0 \\
\hline
\end{tabular}

aNot applicable because population immunity to transmission significantly affected by an indigenous cVDPV outbreak

Table 3: Impact of the annual number of bOPV SIAs on the vulnerability to importations of OPV-related viruses with different degrees of reversion in different populations.

\begin{tabular}{|c|c|c|c|c|c|c|c|c|c|c|c|c|}
\hline \multirow[t]{2}{*}{ Population with properties like } & \multicolumn{3}{|c|}{$\begin{array}{l}\text { Indigenous cVDPV1 } \\
\text { outbreaks before/after } \\
\text { OPV13 cessation }\end{array}$} & \multicolumn{3}{|c|}{$\begin{array}{l}\text { Indigenous cVDPV3 } \\
\text { outbreaks before/after } \\
\text { OPV13 cessation }\end{array}$} & \multicolumn{3}{|c|}{$\begin{array}{c}\text { Percent of time during } \\
\text { 2017-2019 with } \mathrm{Rn}>1 \text { for } \\
\text { cVDPV1 }\end{array}$} & \multicolumn{3}{|c|}{$\begin{array}{l}\text { Percent of time during } \\
\text { 2017-2019 with } \mathrm{Rn}>1 \text { for } \\
\text { cVDPV3 }\end{array}$} \\
\hline & $\mathrm{I}^{\mathrm{a}}$ & $M 1^{b}$ & $M 3^{c}$ & 1 & M1 & M3 & 1 & M1 & M3 & 1 & M1 & M3 \\
\hline Northern India, general & None & None & None & None & None & None & 88 & 63 & 44 & 70 & 40 & 0 \\
\hline Northern India, under-vaccinated & Before & None & None & None & None & None & $N A^{d}$ & 91 & 60 & $94^{\mathrm{e}}$ & 67 & 25 \\
\hline Northern Pakistan and Afghanistan, general & None & None & None & None & None & None & 59 & 28 & 0 & 38 & 0 & 0 \\
\hline Northern Pakistan and Afghanistan, under-vaccinated & Before & After & After & Before & None & None & $N A^{d}$ & 100 & 100 & $97^{\mathrm{e}}$ & 97 & 86 \\
\hline Northern Nigeria, general & None & None & None & None & None & None & 64 & 11 & 0 & 35 & 0 & 0 \\
\hline Northern Nigeria, under-vaccinated & Before & After & None & None & None & None & $N A^{d}$ & 100 & 100 & $100^{\mathrm{e}}$ & 100 & 100 \\
\hline
\end{tabular}

al indicates bOPV intensification consisting of 0 annual bOPV SIAs during 2017-2019 and between 1 and 4 bOPV SIAs in 2020 prior to OPV13 cessation

${ }^{b} \mathrm{M} 1$ indicates bOPV maintenance consisting of 1 annual bOPV SIA during 2017-2020

cM3 indicates bOPV maintenance consisting of 3 annual bOPV SIA during 2017-2020

dNot applicable: Indigenous cVDPV outbreak prior to OPV13 cessation

eSIAs in response to indigenous CVDPV 1 outbreak would affect population immunity to serotype 3 transmission

Table 4: Implications of intensification versus maintenance in terms of indigenous cVDPV outbreaks and vulnerability to WPV or cVDPV importations. 
WPV1 eradication. Moreover, high population immunity to poliovirus transmission at the time of OPV13 cessation extends the time until populations become vulnerable to the transmission of OPV1- and OPV3-related viruses, including serotype 1 and $3 \mathrm{mOPV}$ potentially introduced deliberately to respond to outbreaks.

The occurrence of relatively frequent cVDPV2s in the time period shortly before OPV2 cessation came about as a direct result of national and programmatic decisions to use serotype 1 and $3 \mathrm{mOPV}$ and bOPV for SIAs instead of tOPV. Based on the results of modeling that demonstrated the importance of increasing population immunity to serotype 2 transmission in countries with poorly performing RI, national and global polio program managers intensified tOPV use in SIAs prior to OPV2 cessation which led to increased demand for tOPV $[39,40]$, Ideally, following OPV2 cessation, countries will continue to use bOPV SIAs to maintain population immunity to serotype 1 and 3 transmission between now and the time of OPV13 cessation, which will maintain vaccine demand, support ongoing and predictably high levels of bOPV production, and reduce or eliminate the need to intensify bOPV use prior to OPV13 cessation. Vaccine manufacturers will need to continue bOPV production in sufficient quantities to support global demands, including vaccine for outbreak response SIAs, until OPV13 cessation, and they will also need to also produce serotype 1 and $3 \mathrm{mOPV}$ for outbreak response vaccine stockpiles. This implies that maintaining ongoing support for vaccine purchase represents a critical risk management strategy. In contrast to the situation with OPV2 cessation, for which manufacturers switched from tOPV to bOPV production, after OPV13 cessation manufacturers will cease production of OPV altogether after filling the demand for mOPV to maintain a limited global stockpile for response to poliovirus outbreaks. This represents a fundamentally different change in the manufacturing market, with OPV production facilities decommissioned and/or repurposed after OPV13 cessation.

While the model suggests that countries at risk of cVDPVs after OPV13 cessation could largely cease bOPV SIAs and then pursue a strategy of bOPV intensification in the 6 months prior to OPV13 cessation to still prevent cVDPVs after OPV13 cessation, such a strategy comes with two significant potential unintended consequences. First, if these countries reduce the frequency of bOPV SIAs, then they increase their risk of outbreaks of imported WPV1 and/or cVDPVs prior to OPV13 cessation, which will lead to the need for expensive and unplanned outbreak response SIAs that also requires vaccine doses Countries at risk of cVDPVs will likely substantially reduce risks of serotype 1 and 3 poliovirus outbreaks if they continue to conduct at least one annual bOPV SIA, with the risk of outbreaks decreasing with increasing bOPV SIA frequency. Although this analysis showed that relatively low minimum routine immunization coverage is required to prevent indigenous cVDPV1 and cVDPV3 outbreaks after OPV13 cessation (Figure 1), we emphasize that all communities need to maintain these levels and that, due to heterogeneity within countries, maintaining national average population immunity to transmission at these thresholds will not always suffice to prevent indigenous cVDPVs. In this context, continuing to conduct bOPV SIAs will help maintain population immunity to serotype 1 and 3 transmission in all communities and reduce cVDPV risks. Second, if countries cease bOPV SIAs, reduce their orders for bOPV, and contribute to global reductions in bOPV demand, then some manufacturers may leave the market early, which will reduce global production capacity. This may then lead to insufficient global bOPV production capacity to support bOPV intensification efforts that countries and the global program would want or need to conduct prior to OPV13 cessation. Insufficient population immunity prior to OPV 13 cessation to prevent cVDPVs will ultimately increase the demand for outbreak response resources, including mOPV from the outbreak response stockpile, and lead to the need for increased production of OPV.

This analysis relied on a DEB model with previously discussed limitations related to uncertainty in model inputs and simplification of mixing assumptions for large populations [25]. While the generic model inputs (i.e., those that apply to all populations, like the OPV reversion time) represent the result of extensive expert input and model calibration that reproduced existing evidence $[12,25,29,30]$, we cannot preclude that other combinations of model inputs might similarly or better approximate the uncertain real world values. This analysis also used simplified realistic populations to show the spectrum of possible outcomes and demonstrate the effect of changing population-specific assumptions. Real populations include more heterogeneity and more complicated poliovirus exposure and vaccination histories [29]. While the numerically optimal number of bOPV SIAs will vary between populations, we do not expect substantially different qualitative insights for other, real high-risk populations than those based on the simplified examples presented in this analysis. Our focus on cVDPV emergence defined as virus in the last reversion stage that transmits at the population level means that we did not consider occasional occurrence of viruses from healthy vaccine recipients or their contacts that meet the virological VDPV definition and represent the tail of the distribution of excreted viruses [41]. These events may occur even with sufficient bOPV SIAs but likely remain epidemiologically inconsequential. We also did not consider the implications of bOPV SIA choices on iVDPV and other long-term risks, considered elsewhere [17,34]. However, we believe that our choice of populations adequately approximates population immunity dynamics in real high-risk settings. We hope that our analysis provides helpful context related to the management of serotype 1 and 3 population immunity to transmission until OPV13 cessation.

\section{Conclusions}

Although some reduction in global SIA frequency can safely occur, countries with suboptimal routine immunization coverage should each continue to conduct at least one annual SIA with bOPV, preferably more, until global OPV13 cessation. Preventing cVDPV risks after OPV13 cessation requires investments in bOPV SIAs now through the time of OPV13 cessation. Managing vaccine supply chain issues for bOPV until after bOPV13 cessation remains a critical risk management issue.

\section{Financial Support}

Financial support for this study was provided by the Centers for Disease Control and Prevention (Cooperative Agreement Number 1U2RGH001913-01).

\section{Competing Interests}

None

\section{Acknowledgments}

This publication was supported by Cooperative Agreement Number 1U2RGH001913-01 funded by the Centers for Disease Control and Prevention. Its contents are solely the responsibility of the authors and do not necessarily represent the official views of the Centers for Disease Control and Prevention or the Department of Health and Human Services.

\section{References}

1. World Health Organization (2001) Transmission of wild poliovirus type 2 Apparent global interruption. Wkly Epidemiol Rec 76: 95-97. 
Citation: Duintjer Tebbens RJ, Hampton LM, Wassilak SGF, Pallansch MA, Cochi SL, et al. (2016) Maintenance and Intensification of Bivalent Oral Poliovirus Vaccine Use Prior to its Coordinated Global Cessation. J Vaccines Vaccin 7: 340. doi: 10.4172/2157-7560.1000340

2. Global Polio Eradication Initiative (2015) Global eradication of wild poliovirus type 2 declared.

3. Kew OM, Cochi SL, Jafari HS, Wassilak SG, Mast EE, et al. (2014) Possible eradication of wild poliovirus type 3-worldwide, 2012. MMWR Morb Mortal Wkly Rep 63: 1031-1033.

4. World Health Organization (2005) Polio Eradication Initiative. Cessation of Routine Oral Polio Vaccine (OPV) use after Global Polio Eradication. Framework for National Policy Makers in OPV-using Countries.

5. Duintjer Tebbens RJ, Pallansch MA, Kew OM, Cáceres VM, Jafari H, et al (2006) Risks of paralytic disease due to wild or vaccine-derived poliovirus after eradication. Risk Anal 26: 1471-1505.

6. Global Polio Eradication Initiative (2016) Global synchronisation and the switch.

7. World Health Organization (2013) Global Polio Eradication Initiative: Polio Eradication and Endgame Strategic Plan (2013-2018).

8. Thompson KM, Duintjer Tebbens RJ (2012) Current polio global eradication and control policy options: Perspectives from modeling and prerequisites for OPV cessation. Exp Rev Vaccines 11: 449-459.

9. Thompson KM, Duintjer Tebbens RJ (2015) Health and economic consequences of different options for timing the coordinated global cessation of the three oral poliovirus vaccine serotypes. BMC Infect Dis 15: 376.

10. The Cuba IPV Study Collaborative Group (2007) Randomized, placebocontrolled trial of inactivated poliovirus vaccine in Cuba. N Engl J Med 356 1536-1544.

11. Hird TR, Grassly NC (2012) Systematic review of mucosal immunity induced by oral and inactivated poliovirus vaccines against virus shedding following oral poliovirus challenge. PLoS Pathog 8: e1002599.

12. Duintjer Tebbens RJ, Pallansch MA, Chumakov KM, Halsey NA, Hovi T, et al (2013) Expert review on poliovirus immunity and transmission. Risk Anal 33 544-605.

13. Thompson KM, Duintjer Tebbens RJ (2014) National choices related to inactivated poliovirus vaccine, innovation, and the end game of global polio eradication. Exp Rev Vaccines 13: 221-234.

14. Duintjer Tebbens RJ, Thompson KM (2014) Modeling the potential role of inactivated poliovirus vaccine to manage the risks of oral poliovirus vaccine cessation. J Infect Dis 210: S485-S497.

15. Kalkowska DA, Duintjer Tebbens RJ, Thompson KM (2014) Modeling strategies to increase population immunity and prevent poliovirus transmission in two high-risk areas in northern India. J Infect Dis 210: S398-S411.

16. Kalkowska DA, Duintjer Tebbens RJ, Thompson KM (2014) Modeling strategies to increase population immunity and prevent poliovirus transmission in the high-risk area of northwest Nigeria. J Infect Dis 210, S412-S423.

17. Duintjer Tebbens RJ, Pallansch MA, Thompson KM (2015) Modeling the prevalence of immunodeficiency-associated long-term vaccine-derived poliovirus excretors and the potential benefits of antiviral drugs. BMC Infect Dis 15: 379

18. Onorato IM, Modlin JF, McBean MA, Thoms ML, Losonsky GA, et al. (1991) Mucosal immunity induced by enhanced-potency inactivated and oral polio vaccines. $J$ Infect Dis 163: 1-6.

19. Thompson KM, Pallansch MA, Duintjer Tebbens RJ, Wassilak SGF, Cochi SL (2013) Modeling population immunity to support efforts to end the transmission of live polioviruses. Risk Anal 33: 647-663.

20. Thompson KM, Duintjer Tebbens RJ (2014) Modeling the dynamics of ora poliovirus vaccine cessation. J Infect Dis 210: S475-S484.

21. Kalkowska DA, Duintjer Tebbens RJ, Pallansch MA, Cochi SL, Wassilak SGF, et al. (2015) Modeling undetected live poliovirus circulation after apparent interruption of transmission: Implications for surveillance and vaccination. BMC Infect Dis 15: 1.

22. Duintjer Tebbens RJ, Hampton LM, Thompson KM (2016) Implementation of coordinated global serotype 2 oral poliovirus vaccine cessation: Risks of potential non-synchronous cessation. BMC Infect Dis 16: 237

23. Duintjer Tebbens RJ, Hampton LM, Thompson KM (2016) Implementation of coordinated global serotype 2 oral poliovirus vaccine cessation: Risks of inadvertent trivalent oral poliovirus vaccine use. BMC Infect Dis 16: 231

24. Duintjer Tebbens RJ, Pallansch MA, Wassilak SGF, Cochi SL, Thompson KM (2016) Characterization of outbreak response strategies and potential vaccine stockpile needs for the polio endgame. BMC Infect Dis 16: 137.

25. Duintjer Tebbens RJ, Pallansch MA, Kalkowska DA, Wassilak SG, Cochi SL, et al. (2013) Characterizing poliovirus transmission and evolution: Insights from modeling experiences with wild and vaccine-related polioviruses. Risk Anal 23 703-749.

26. Duintjer Tebbens RJ, Kalkowska DA, Wassilak SGF, Pallansch MA, Cochi SL, et al. (2014) The potential impact of expanding target age groups for polio immunization campaigns. BMC Infect Dis 14: 45.

27. Kalkowska DA, Duintjer Tebbens RJ, Grotto I, Shulman LM, Anis E, et al. (2015) Modeling options to manage type 1 wild poliovirus imported into Israel in 2013. J Infect Dis 211: 1800-1812.

28. Diekmann O, Heesterbeek JA, Metz JA (1990) On the definition and the computation of the basic reproduction ratio $\mathrm{R} 0$ in models for infectious diseases in heterogeneous populations. J Math Biol 28: 365-382.

29. Duintjer Tebbens RJ, Pallansch MA, Chumakov KM, Halsey NA, Hovi T, et al. (2013) Review and assessment of poliovirus immunity and transmission: Synthesis of knowledge gaps and identification of research needs. Risk Anal 33: 606-646.

30. Duintjer Tebbens RJ, Pallansch MA, Kim JH, Burns CC, Kew OM, et al. (2013) Review: Oral Poliovirus Vaccine Evolution and Insights Relevant to Modeling the Risks of Circulating Vaccine-Derived Polioviruses (cVDPVs). Risk Anal 23 680-702.

31. Kew O, Morris-Glasgow V, Landaverde M, Burns C, Shaw J, et al. (2002) Outbreak of poliomyelitis in Hispaniola associated with circulating type vaccine-derived poliovirus. Science 296: 356-359.

32. Kew OM, Sutter RW, de Gourville EM, Dowdle WR, Pallansch MA (2005) Vaccine-derived polioviruses and the endgame strategy for global polio eradication. Ann Rev Microbiol 59: 587-635.

33. Burns C, Diop O, Sutter RW, Kew OM (2014) Vaccine-derived polioviruses. J Infect Dis 210: S283-S293.

34. Duintjer Tebbens RJ, Pallansch MA, Cochi SL, Wassilak SGF, Thompson KM (2015) An economic analysis of poliovirus risk management policy options for 2013-2052. BMC Infect Dis 15: 389.

35. World Health Organization (2014) WHO/UNICEF estimated coverage time series. WHO and UNICEF.

36. Benyesh-Melnick M, Melnick JL, Rawls WE, Wimberly I, Barrera Ora J, et al (1967) Studies of the immunogenicity, communicability and genetic stability of oral poliovaccine administered during the winter. Am J Epidemiol 86: 112-136.

37. Chen RT, Hausinger S, Dajani AS, Hanfling M, Baughman AL, et al. (1996) Seroprevalence of antibody against poliovirus in inner-city preschool children. JAMA 275: 1639-1645.

38. Thompson KM, Kalkowska DA, Duintjer Tebbens RJ (2015) Managing population immunity to reduce or eliminate the risks of circulation following the importation of live polioviruses. Vaccine 33: 1568-1577.

39. Duintjer Tebbens RJ, Thompson KM (2015) Managing the risk of circulating vaccine-derived poliovirus during the endgame: Oral poliovirus vaccine needs. BMC Infect Dis 15: 390.

40. UNICEF (2015) Oral polio vaccine supply outlook - December 2015

41. van der Sanden S, Pallansch MA, van de Kassteele J, El-Sayed N, Sutter RW et al. (2009) Shedding of vaccine viruses with increased antigenic and genetic divergence after vaccination of newborns with monovalent type 1 oral poliovirus vaccine. J Virol 83: 8693-8704. 